\title{
Large Seebeck Coefficients of Protonated Titanate Nanotubes for High-Temperature Thermoelectric Conversion
}

\author{
L. Miao,${ }^{*}{ }^{\dagger}$ S. Tanemura, ${ }^{\dagger,}{ }^{\dagger}$ R. Huang, ${ }^{\S}$ C.Y. Liu, ${ }^{\dagger}$ C.M. Huang, ${ }^{\dagger}$ and G. $\mathrm{Xu}^{\dagger}$
}

Key Laboratory for Renewable Energy and Gas Hydrates, Guangzhou Institute of Energy Conversion, Chinese Academy of Sciences, No. 2 Nengyuan Road, Tianhe District, Guangzhou, 510640 P.R. China, Nanostructures Research Laboratory, Japan Fine Ceramics Center, 2-4-1 Mutsuno, Atsuta-ku, Nagoya, 456-8587, Japan, and Key Laboratory of Polar Materials and Devices, Ministry of Education, East China Normal University, No. 3663 North Zhongshan Road, Putuo District, Shanghai 200062, P.R. China

\begin{abstract}
Titanate nanotubes $\mathrm{Na}_{2-x} \mathrm{H}_{x} \mathrm{Ti}_{3} \mathrm{O}_{7}$ produced by alkali hydrothermally treated ground $\mathrm{TiO}_{2}$ aerogels are investigated as possible materials for high-temperature thermoelectric conversion by measuring their thermoelectric properties. Strikingly, the Seebeck coefficients increased sharply in the temperature range 745 to $1032 \mathrm{~K}$, reaching a maximum of $302 \mu \mathrm{V} / \mathrm{K}$. The electrical resistivity of the TNNTs ranged from 325 to $525 \Omega \mathrm{m}$, which is lower than that of bulk $\mathrm{TiO}_{2}$, and thermal conductivities at room temperature were also very low, ranging from 0.55 to $0.75 \mathrm{Wm}^{-1} \mathrm{~K}^{-1}$. The hollow structure of the titanate nanotubes, with small, uniform diameters, is thought to be responsible for the ultralow thermal conductivity. The large thermoelectric power and ultralow thermal conductivity suggest that titanate nanotubes represent a new kind of p-type oxide thermoelectric material.
\end{abstract}

KEYWORDS: thermoelectric conversion $\bullet$ Seebeck coefficients $\bullet$ titanate nanotubes $\bullet$ oxide nanomaterials

\section{INTRODUCTION}

hermoelectric (TE) devices can convert heat originat-
ing from various sources, for example, solar thermal,
geothermal, electric power plants, factories, automobiles, computers, and even human bodies to electric power using the Seebeck effect, in addition to being able to refrigerate various devices by means of the Peltier effect $(1,2)$. Such devices are environmentally friendly in the sense that they produce essentially no waste material. The performance of TE materials is evaluated in terms of a dimensionless figure of merit, $Z T$, where $Z T=S^{2} \sigma T \kappa^{-1}$, and $Z, T, S, \sigma$, and $\kappa$ are, respectively, the figure of merit, the absolute temperature, the thermopower or Seebeck coefficient, the electrical conductivity, and the thermal conductivity. Highperformance thermoelectric materials currently constitute one of the main areas of materials research for environmentally friendly energy conversion technologies driven by the need to develop low $\mathrm{CO}_{2}$ emissions technology.

A good thermoelectric material should have a relatively high thermopower (Seebeck coefficient). Because there is no theoretical upper limit of $S$, it can be, in principle, as efficient as any other energy-conversion system. In addition, the lifetime of the device can be as long as the lifetime of the material from which the device is made. Hence, the search

* Corresponding author. E-mail: miaolei@ms.giec.ac.cn. Tel. and Fax: +86-20-8703-5351.

Received for review April 27, 2010 and accepted July 14, 2010

${ }^{\dagger}$ Chinese Academy of Sciences

* Japan Fine Ceramics Center.

$\S$ East China Normal University.

DOI: 10.1021/am100365y

2010 American Chemical Society for a new kind of high-performance TE material is still very urgent, especially for high-temperature conversion. Over the last three decades, $\mathrm{Si}-\mathrm{Ge}$ alloys (3), several transition-metal chalcogenides (4), transition-metal disilicides (5), and some boron compounds $(6,7)$ have been developed as materials for high-temperature thermoelectric power generation. However, practical utilization has been limited because many of these materials require costly surface protection to prevent oxidation or vaporization, and others have inherent temperature limits owing to phase transitions at high temperature. With respect to high-temperature operation in air, metal oxides in their common oxidation state have clear advantages because of their excellent stability to heat.

As is well-known, it is hard to achieve high ZT in traditional bulk materials because of the Wiedmann-Franz law. Recently, both theoretical analysis and experimental results have shown that low-dimensional nanostructures can enhance the Seebeck coefficient greatly $(8-10)$. Nanocomposite and nanoporous materials are used in a variety of applications with rapid progress being made in the controlled synthesis of nanowires, nanotubes, and nanoparticles $(11,12)$. Unfortunately, although the most widely used commercial thermoelectric materials are bulk $\mathrm{Bi}_{2} \mathrm{Te}_{3}$ and its alloys with $\mathrm{Sb}$, Se, and so on, fabricating synthetic $\mathrm{Bi}_{2} \mathrm{Te}_{3}$ nanostructures is difficult and expensive (10).

Recently, several metal oxides with layered structure and strongly correlated electronic system, including $\mathrm{SrTi}_{0.8^{-}}$ $\mathrm{Nb}_{0.2} \mathrm{O}_{3},\left(Z T_{300 \mathrm{~K}} \sim 0.08, Z T_{1000 \mathrm{~K}}=0.37\right)(11,12)$, Nb-doped $\mathrm{TiO}_{2}, \mathrm{Na}_{0.7} \mathrm{CoO}_{2}\left(Z T_{300 \mathrm{~K}} \sim 0.1\right)(13)$, and $\mathrm{Ca}_{3} \mathrm{CO}_{4} \mathrm{O}_{9}\left(Z T_{300 \mathrm{~K}}\right.$ $\left.\sim 0.08, Z T_{1000 \mathrm{~K}} \sim 0.3\right)$ (14), have been synthesized that exhibit a rather high $Z T$. These metal oxides have attracted 
increasing attention as sources for generating thermoelectric power at high temperatures because they have potential advantages over state-of-the-art, high temperature thermoelectric materials, including a SiGe-based alloy $(Z T \sim 1)(15)$ and $\beta-\mathrm{FeSi}_{2}(Z T \sim 0.3)$ (16), in terms of chemical and thermal resistances at high temperatures $(1000 \mathrm{~K})$. One of the most important features of these metal oxides as TE materials are rather large Seebeck coefficient $|S|$ even in a heavily carrier doped state because strongly correlated electrons system occurred in layered metal-oxide structure.

Protonated titanate nanotubes (TNNT) synthesized by the hydrothermal method exhibit excellent properties for use in applications such as hydrogen storage $(17-20)$, sensors $(21,22)$, batteries (23), electrochromism (24), photoluminescence (25), and supporting matrices for proteins (26). A major advantage of titanate nanotubes is their easy and lowcost fabrication. The unit cell of a titanate nanotube comprises $\mathrm{TiO}_{6}$ octahedra similar to those of $\mathrm{SrTiO}_{3}$ and $\mathrm{La}_{1-x} \mathrm{Y}_{x} \mathrm{TiO}_{3}$. Furthermore, the layered structure of multiwall nanotubes is similar to that of alternately layered metal oxides such as $\mathrm{Na}_{0.7} \mathrm{CoO}_{2}$, although the spacing and stacking number is different. The proton and sodium ions existing between multiwall interlayers in the nanotubes contribute to novel optical and electrical properties.

In a series of previous papers $(27,28,30)$, properties of the synthesized nanotubes such as composition, morphology, crystal structure, chirality, and rolling-up mechanism have been reported. Unexpectedly, we found that in the case of $\mathrm{H}, \mathrm{Na}-(\mathrm{H}, \mathrm{Na}) \mathrm{TNNTS}$ the Fermi level in the Ti band gap is close to the bottom of the conductance band (28), which may be related to the enhancement of the Seebeck coefficient. Motivated by this finding, we carried out an investigation of the thermoelectric properties of $(\mathrm{H}, \mathrm{Na}) \mathrm{TNNTs}$ which we report here.

\section{EXPERIMENTAL SECTION}

2.1. Materials and Methods. All chemicals were used as received. Deionized water was used in all of the aqueous solutions and washings. Titanate nanotubes with high aspect ratios, large specific surface areas and uniform dispersion were produced by alkaline hydrothermal treatment of ground $\mathrm{TiO}_{2}$ aerogels followed by freeze-drying.

2.2. Synthesis of $\mathrm{TiO}_{2}$ Aerogels. Aerogels produced by this sol-gel and supercritical drying method have several significant advantages: large surface area $\left(\sim 1000 \mathrm{~m}^{2} / \mathrm{g}\right)$, low density $(\sim 0.1$ $\mathrm{g} / \mathrm{cm}^{3}$ ) and high porosity ( $\sim 98 \%$ ), leading to the formation of high quality nanotubes. We first synthesize $\mathrm{TiO}_{2}$ wetgels using titanium tetra-isopropoxide ( $\mathrm{Ti}(\mathrm{PrOH})_{4}$ or TTIP), ethanol (EtOH), water and nitric acid by a sol-gel method. After aging, gelation, and rinsing, $\mathrm{TiO}_{2}$ wetgels were supercritical dried using $\mathrm{CO}_{2}$ at $9.5 \mathrm{MPa}$ and $373 \mathrm{~K}$ for $2 \mathrm{~h}$ to form $\mathrm{TiO}_{2}$ aerogels

2.3. Synthesis of Titanate Nanotubes. The ground $\mathrm{TiO}_{2}$ aerogels were hydrothermally treated using $10 \mathrm{M} \mathrm{NaOH}$ solution at $150{ }^{\circ} \mathrm{C}$ for $40 \mathrm{~h}$ in a stainless Teflon-lined autoclave. Details of the fabrication process are reported elsewhere $(27,28)$. Recently, the importance of strong alkaline conditions during the fabrication process has been justified by Mori et al. (29) using theoretical methods. Freeze-drying was carried out in a Christ Alpha 2-4 freeze-dryer and precisely controlled by selecting an appropriate solvent. Vacuum drying was performed at $10 \mathrm{~Pa}$ and $100^{\circ} \mathrm{C}$ for $10 \mathrm{~h}$ in a horizontal tube furnace.

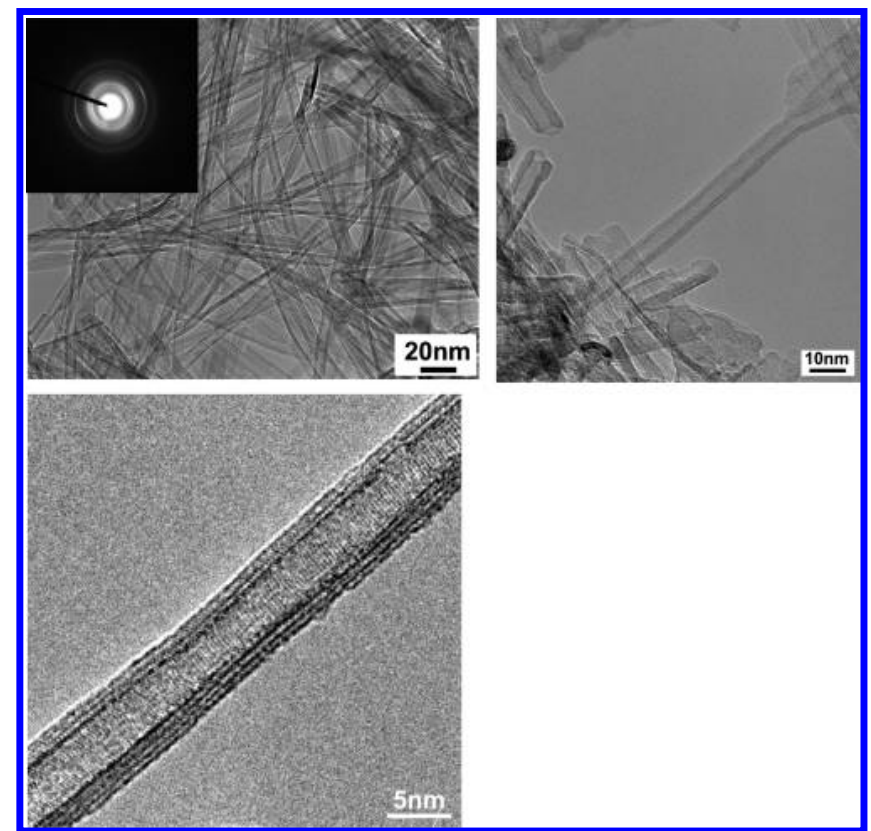

FIGURE 1. TEM images of (a) titanate nanotubes at low magnification and the corresponding electron diffraction pattern; (b) a single nanotube at low magnification; (c) a single nanotube at high magnification.

The nanotubes made from ground $\mathrm{TiO}_{2}$ aerogel powder precursors possess larger specific surface area $\left(305 \mathrm{~m}^{2} / \mathrm{g}\right)$ compared with those made from commercial available $\mathrm{TiO}_{2}$ powder $\left(176 \mathrm{~m}^{2} / \mathrm{g}\right)$. Moreover, the nanotubes produced by this method show good crystal quality, uniform dispersion and small cross-sectional diameter. As will be demonstrated in the following section, all of these characteristics contribute to improved TE performance.

The nanotubes produced by this hydrothermal method have no particular orientation and are randomly distributed throughout the obtained powder. For Seebeck coefficient and resistivity measurements, the as-prepared powder was cold-pressed into a cylindrical pellet $3 \mathrm{~mm}$ in diameter and $6 \mathrm{~mm}$ thick using an axial pressure of ca. $0.43 \mathrm{MPa}$. The density of the pellet was $3.04 \mathrm{~g} / \mathrm{cm}^{3}$. For the measurement of thermal conductivity, the as-prepared powder was cold-pressed into a cylindrical pellet $10 \mathrm{~mm}$ in diameter and $2 \mathrm{~mm}$ in thickness. Given the random distribution of nanotubes in the powder, the physical properties of the pellet were assumed to be isotropic.

2.4. Instruments and Characterization. The morphology and structure of nanotubes were observed by transmission electron microscopy (TEM) using a JEOL, JEM-3000F microscope at $300 \mathrm{kV}$. The temperature dependence of Seebeck coefficients and resistivity of $(\mathrm{H}, \mathrm{Na})$ TNNTs on the macroscopic scale were evaluated using a ZEM-3 Seebeck coefficient/electrical resistance measuring system (ULVAC-RIKO) in the temperature range $273-1073 \mathrm{~K}$. Thermal conductivities of the samples were measured using an SPTM-1000 scanning thermal probe microimage system (ULVAC-RIKO) and a laser flash (LF) thermal constants analyzer (ULVAC-RIKO) on micro- and macroscopic scales, respectively.

\section{RESULTS AND DISCUSSION}

3.1. Morphology and Composition. Figure 1 shows TEM micrographs of the morphology of the fabricated nanotubes at low and high magnifications, revealing their uniform dispersion and fine structure. When ground $\mathrm{TiO}_{2}$ aerogels are used as precursor material instead of commercially available powders, the nanotubes show good size 


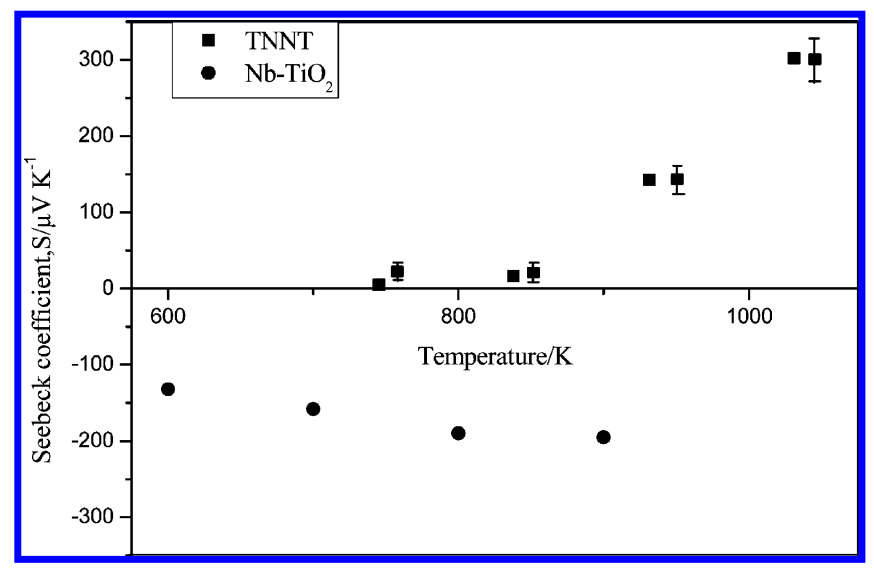

FIGURE 2. Temperature dependence of Seebeck coefficient for titanate nanotubes. $S$ values from ref (31) are also included.

uniformity with inner and outer diameters of 4-7 and 10-11 nm, respectively, and lengths of several hundreds of nanometers. Detailed analyses of the composition, morphology, crystal structure, chirality, and rolling-up mechanism of the $\mathrm{TiO}_{6}$ octahedron nanosheets of the nanotubes have been reported previously $(27,28,30)$. In particular, the presence of sodium ions $\left(\mathrm{Na}^{+}\right)$and the intercalated layered structure of titanate nanotubes have been identified by X-ray diffraction (XRD), Raman spectroscopy as well as energydispersive X-ray spectroscopy (EDX) as given in previous publications $(28,30)$. The position and bonding states of alkali ions and protons are different. $\mathrm{Na}^{+}$ions are located between titanate layers at a distance of more than $2 \AA$ from the nearest oxygen ion, while the distance between a proton $\left(\mathrm{H}^{+}\right)$and the nearest octahedral oxygen ion is $1 \AA$, which is significantly shorter and implies covalent bonding. The weaker bonding of $\mathrm{Na}^{+}$ion suggests the possibility of ion exchange.

3.2. Seebeck Coefficients. The temperature dependence of the Seebeck coefficients is shown in Figure 2. Because of the poor electrical conductivity of the pressed TNNT pellet at low temperatures, both Seebeck coefficients and resistivity values were measured from 745 to $1032 \mathrm{~K}$. The Seebeck coefficients increased sharply in this temperature range, reaching a maximum of $302 \mu \mathrm{V} / \mathrm{K}$. The $S$ values of the $(\mathrm{H}, \mathrm{Na}) \mathrm{TNNTS}$ are positive over the whole temperature range, indicating $\mathrm{p}$-type conduction.

Compared with values for $\mathrm{TiO}_{2}$ thin film (31), also plotted in Figure 2, protonated ( $\mathrm{H}, \mathrm{Na}$ )TNNTs exhibit a large absolute value of $S$, indicating good high-temperature thermoelectric conversion. Because $\mathrm{TiO}_{2}$ is a typical n-type semiconductor, Nb-doped $\mathrm{TiO}_{2}$ thin films showed similar n-typed behaviors. Although the $\left(\mathrm{H}\right.$, Na)TNNTs are formed from $\mathrm{TiO}_{2}$ raw materials, the p-type semiconductor behavior may be related to the intercalated sodium and hydrogen ions present between the multiwall layers (32).

One explanation for the large Seebeck coefficient of protonated titanate nanotubes $\mathrm{Na}_{2-x} \mathrm{H}_{x} \mathrm{Ti}_{3} \mathrm{O}_{7}$ may be the carrier effective mass, $\mathrm{m} * \mathrm{~m}_{0}$. The effective mass is one of the main factors determining the Seebeck coefficient and electrical conductivity of thermoelectrics. As is known, $\mathrm{TiO}_{2}$ semiconductors have a large effective mass both for elec-

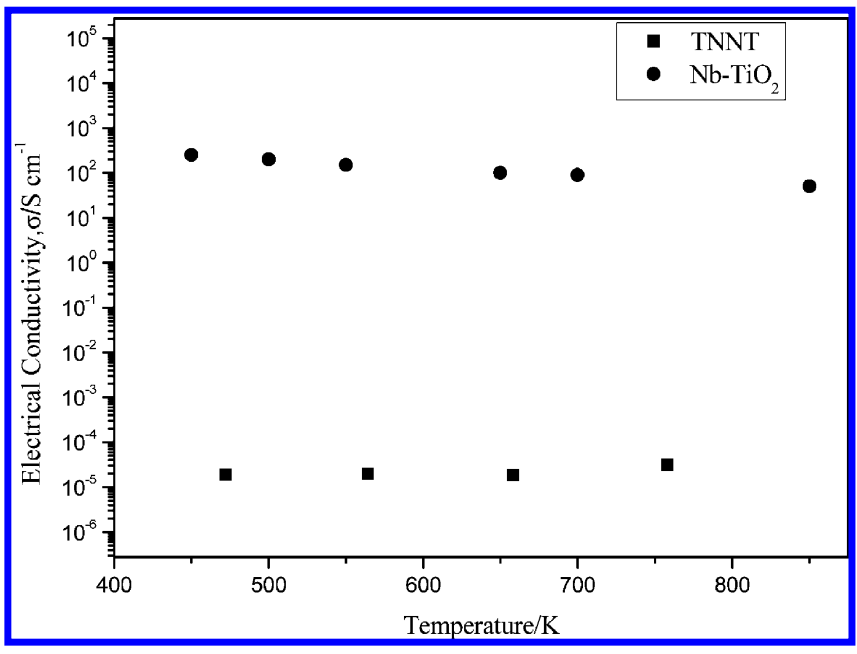

FIGURE 3. Temperature dependence of electrical conductivity for titanate nanotubes $\sigma$ values from ref (31) are also included.

Table 1. Comparison of Thermal Conductivities of $(\mathrm{H}, \mathrm{Na}) \mathrm{TNNTs}$ and Bulk $\mathrm{TiO}_{2}$

\begin{tabular}{lcc}
\hline & $(\mathrm{H}, \mathrm{Na}) \mathrm{TNNTs}$ & bulk $\mathrm{TiO}_{2}$ \\
\hline thermal conductivity $(\mathrm{W} / \mathrm{mK})$ & $\begin{array}{l}0.55^{a} \\
0.75^{b}\end{array}$ & $8.4(31)$.
\end{tabular}

${ }^{a}$ SPTM (microscopic). ${ }^{b}$ LF (macroscopic).

trons $\left(m_{\mathrm{e}} * / m_{0}=9\right)$ and holes $\left(m_{\mathrm{h}}{ }^{*} / m_{0}=3\right)(33)$. The effective electron mass changes from 0.802 for pure $\mathrm{SrTiO}_{3}$ to 15 for doped thermoelectric materials (34). Although no experimental or theoretical carrier effective masses have so far been reported, titanate nanotubes are likely to exhibit enhanced carrier effective masses compared to $\mathrm{TiO}_{2}$ partly because of the intercalation of sodium ions, leading to an increased lattice parameter. Another reason for the large Seebeck coefficient may be related to the elevated Fermi level, which reaches almost to the bottom of conductance band, as determined by energy loss near edge structure (ELNES) spectra (30).

3.3. Electrical and Thermal Conducitivity. The electrical conductivity of $(\mathrm{H}, \mathrm{Na}) \mathrm{TNNTS}$ increases rapidly as temperature is increased, as shown in Figure 3. The electrical conductivity of $(\mathrm{H}, \mathrm{Na})$ TNNTs is much lower than that reported for $\mathrm{Nb}-\mathrm{TiO}_{2}$ (31), but greater than that of bulk $\mathrm{TiO}_{2}$ (35). The electrical resistivity of the $(\mathrm{H}, \mathrm{Na})$ TNNTs ranged from 325 to $525 \Omega \mathrm{m}$, which is lower than that of bulk $\mathrm{TiO}_{2}$. There are two possible reasons for the relatively low electrical resistivity of TNNT. The first originates from the distortion of $\mathrm{TiO}_{6}$ octahedra, as identified previously (28). The second originates from the intercalated protons existing between multiwalls of the $(\mathrm{H}, \mathrm{Na}) \mathrm{TNNTS}$. One way to further reduce the electrical resistivity of $(\mathrm{H}, \mathrm{Na}) \mathrm{TNNT}$ is ion exchange.

One notable characteristic of TNNT pellets is their ultralow thermal conductivity, as determined by both microand macroscopic measurements, the results of which are listed in Table 1

Compared to those of $\mathrm{TiO}_{2}$ powders (36), the thermal conductivities of $(\mathrm{H}, \mathrm{Na}) \mathrm{TNNTS}$ measured at room temperature are $\mathrm{W} \mathrm{m}^{-1} \mathrm{~K}^{-1}$ (SPTM) and $0.75 \mathrm{Wm}^{-1} \mathrm{~K}^{-1}$ (LF). The hollow tube channels have a strong phonon scattering effect 
similar to that of the cages in a holey solid; one-dimensional nanotubes and two-dimensional tube walls reduce the lattice thermal conductivity further due to the phonon blocking effect of their low-dimensionality (36). These structural features result in $(\mathrm{H}, \mathrm{Na}) \mathrm{TNNTs}$ having low thermal conductivity, making them good candidates for TE conversion. The discussion on temperature dependences of thermal conductivity is omitted here because no significant change of $\mathrm{TiO}_{2}$ thermal conductivity was found in the temperature range 300-1100 K (36).

According to the Wiedemann-Franz law

$$
\begin{gathered}
\kappa_{\text {total }}=\kappa_{\text {lattice }}+\kappa_{\text {electron }} \\
\kappa_{\text {electron }}=L \sigma T
\end{gathered}
$$

where $\kappa_{\text {lattice, }}, \kappa_{\text {electron }}$, and $L$ are thermal conductivity due to lattice and electronic contributions and the Lorentz number respectively. A value of $2.42 \times 10^{-8} \mathrm{~V}^{-2} \mathrm{~K}^{-2}$ was chosen for $L$, corresponding to that of an oxide nondegenerate semiconductor with low carrier doping, based on reported Lorentz numbers for thermoelectric materials $\mathrm{Nb}$-doped $\mathrm{SrTiO}_{3}(11)$, Se-doped $\mathrm{Bi}-\mathrm{Te}$ systems (37), and of $\mathrm{Bi}-\mathrm{Te}$ nanotubes (38). Using the resistivity data shown in Figure 3, we calculated $\kappa_{\text {electron }}$ to be less than $8 \times 10^{-8} \mathrm{~W} / \mathrm{mK}$ at $1032 \mathrm{~K}$ and $4 \times$ $10^{-8} \mathrm{~W} / \mathrm{mK}$ at $930 \mathrm{~K}$. This means that the total thermal conductivity, which is much lower than that reported for polycrystalline $\mathrm{TiO}_{2}$ (36), even after taking into account the fact that the total thermal conductivity experimentally obtained at a room temperature decreases by about $41-43 \%$ compared to the high temperature thermal conductivity, as is widely known to occur for $\mathrm{TiO}_{2}$ polycrystalline (36), is mostly (>about $97 \%$ ) contributed by the lattice, e.g., phonons. The results indicate that the nanostructure leads to a remarkable reduction in thermal conductivity while hardly affecting the electrical transport.

Yang et al. (39) developed a generic model to study phonon transport and thermal conductivity of periodic twodimensional nanocomposites. Their simulation results suggest that composites containing tubular nanowires inclusions have lower effective thermal conductivity than simple nanowire composites because of the introduction of additional surface scattering through the pores associated with the tubular structure. Furthermore, the smaller the pore diameter, the smaller the thermal conductivity is for a given volume fraction. The small and uniform inner diameter (4 $\mathrm{nm})$ of the $(\mathrm{H}, \mathrm{Na})$ TNNTs produced by our hydrothermal method is thus expected to result in significantly lower thermal conductivities.

\subsection{Thermoelectric Conversion Efficiency. Be-} cause of the high electrical resistivity of wide band semiconductor $\mathrm{TiO}_{2}$, little attention was paid to its thermoelectric behavior until Ohta et al. reported measurements on $\mathrm{Nb}$ doped anatase (31). Following the pioneering research of Ohta et al. $(11,31,40)$, titanate compounds have attracted increased attention for TE power applications at high tem-

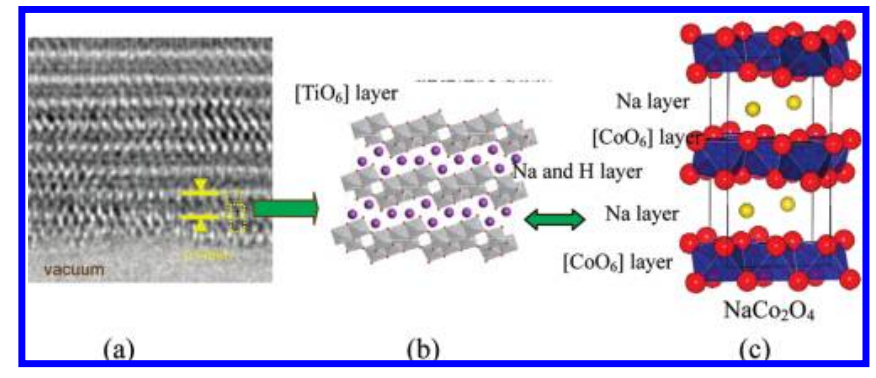

FIGURE 4. Comparison of the stacked $(\mathrm{Na}, \mathrm{H})\left[\mathrm{TiO}_{6}\right]$ layered structure with that of (Na)[CoO 6 ; (a) HRTEM image of a nanotube wall; (b) model of a sodium intercalated triple-wall of a TNNT; (c) crystal structure of TE material $\mathrm{NaCO}_{2} \mathrm{O}_{4}$.

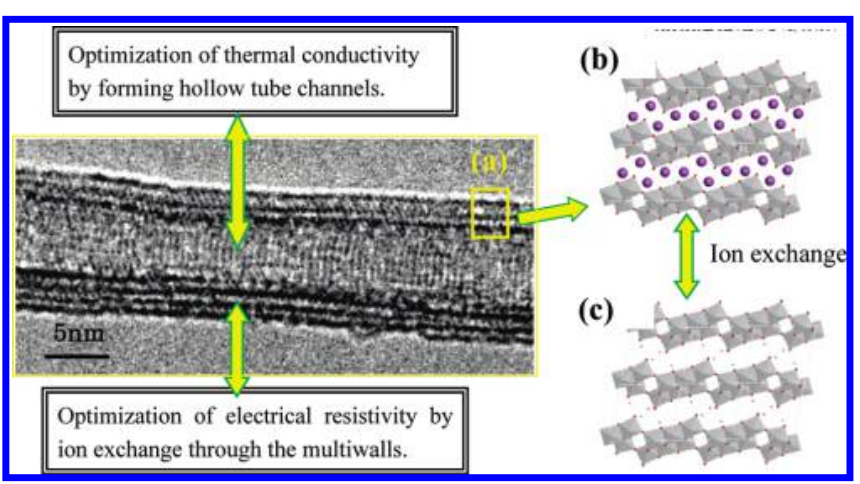

FIGURE 5. Concepts for controlling the electrical conductivity and thermal conductivity based on the special features of titanate nanotubes: (a) HRTEM image of a single nanotube; (b) model of a sodium intercalated triple-wall of a TNNT; (c) model of an ionexchanged triple-wall of a TNNT.

peratures in place of heavy-metal alloys because of their improved chemical and thermal robustness (40). The advantageous features of titanate nanotubes $\mathrm{Na}_{2-x} \mathrm{H}_{x} \mathrm{Ti}_{3} \mathrm{O}_{7}$ include large Seebeck coefficient, controllability of conductivity type (p-or n-type), and the ultralow thermal conductivity resulting from their hollow channels.

A comparsion of stacked $(\mathrm{Na}, \mathrm{H})\left[\mathrm{TiO}_{6}\right]$ layered structure with that of $(\mathrm{Na})\left[\mathrm{CoO}_{6}\right]$ was given in Figure 4. Similar layered structure is found for the two types of materials although further theortical investigation to reveal the evidence of the tail of strongly correlated electron system in sodium titanate nanotube is essentially required. But the comparsion indicates that the potential thermoelectric property of layered titanate compounds.

The figure of merit of the TNNT sample is low because of the poor electrical transport. One way to improve electrical conductivity and achieve high ZT values is the intercalation of ions between the walls of the nanotubes through an ion exchange optimization process, as illustrated in Figure 5. For example, Riss et al. (41) proposed using facile ion exchange in titanate nanostructures to control their photoelectronic properties. The accessibility of the interlayer region to various ions provides an efficient means for controlling the physical and chemical properties of the nanotubes. When an $\mathrm{Na}^{+}$vacancy is introduced, one electron is left in the cell. The excess electrons are localized at the $\mathrm{Na}^{+}$vacancy, so that the $\mathrm{Na}^{+}$vacancy attracts some more electrons from $\mathrm{TiO}_{6}$ layer. On the basis of theoretical calculations, we can select suitable ions for inserting into $\mathrm{TiO}_{6}$ octahedra, thus adjusting 
the carrier mobile, carrier type, carrier effective mass, and density of states and achieving high-performance thermoelectric materials. Because ion intercalation is a very different process from that of cation substitution into $\mathrm{TiO}_{6} \mathrm{sub}$ units, we expect that an increase in electrical resistivity and decrease in thermal conductivity in $(\mathrm{H}, \mathrm{Na}) \mathrm{TNNTs}$ can be achieved simultaneously using this strategy.

\section{CONCLUSIONS AND SUMMARIES}

We successfully synthesized titanate nanotubes of composition $\mathrm{Na}_{2-x} \mathrm{H}_{x} \mathrm{Ti}_{3} \mathrm{O}_{7}$ from alkali-hydrothermally treated ground $\mathrm{TiO}_{2}$ aerogels followed by freeze-drying. The nanotubes have high specific surface area, good crystallinity, and are uniformly dispersed with small cross-sectional diameters. A large p-type Seebeck coefficient of $302 \mu \mathrm{V} / \mathrm{K}$ over the temperature range 745 to $1032 \mathrm{~K}$, ultralow thermal conductivity of 0.55 to $0.75 \mathrm{~W} \mathrm{~m}^{-1} \mathrm{~K}^{-1}$, and high electrical resistivity of 325 to $525 \Omega \mathrm{m}$ were achieved. Our titanate nanotubes, with large carrier effective mass, hollow $1 \mathrm{D}$ structure and 2D multiwalls, are good candidates for $\mathrm{p}$-type high temperature thermoelectric materials.

Acknowledgment. This work was supported by the Guangdong Natural Science Foundation, Grant 9151007006000009 and Guangdong Provincial Science and Technology Grant 2009B010900047. The authors thank Dr. Craig Fisher, Japan Fine Ceramics Center, for helpful discussions.

\section{REFERENCES AND NOTES}

(1) Tritt, T. M.; Subramanian, M. A. MRS Bull. 2006, 31, 188.

(2) DiSalvo, F. I. Science 1999, 285, 703.

(3) Koga, T.; Sun, X.; Cronin, S. B.; Dresselhaus, M. S. Appl. Phvs. Lett. 1999, 75, 2438

(4) Nakahara, J. F.; Takeshita, T.; Tschetter, M. J.; Beaudry, B. J.; Gschneidner, K. A. L. Appl. Phvs. 1988, 63, 2331.

(5) Nishida, I. Phvs. Rev. B 1973, 7, 2710.

(6) Wood, C.; Emin, D. Phvs. Rev. B 1984, 29, 4582.

(7) Yugo, S.; Sato, T.; Kimura, T. Appl. Phvs. Lett. 1985, 46, 842.

(8) Harman, T. C.; Taylor, P. J.; Walsh, M. P.; LaForge, B. E. Science 2002, 297, 2229

(9) Chen, G.; Dresselhaus, M. S.; Dresselhaus, G.; Fleurial, J. P.; Caillat, T. Int. Mater. Rev. 2003, 48, 45

(10) Hochbaum, A. I.; Chen, R.; Delgado, R. D.; Liang, W.; Garnett, E. C.; Najarian, M.; Majumdar, A.; Yang, P. Nature 2008, 451, 163.

(11) Ohta, S.; Nomura, T.; Ohta, H.; Koumoto, K. L.Appl. Phvs. 2005, 97, 034106

(12) Okuda, T.; Nakanishi, K.; Miyasaka, S.; Tokura, Y. Phvs. Rev. B 2001, 63, 113104
(13) Terasaki, I.; Sasago, Y.; Uchinokura, K. Phvs. Rev. B 1997, 56, R12685.

(14) Shikano, M.; Funahashi, R. Appl. Phvs. Lett. 2003, 82, 1851.

(15) Cook, B. A.; Harringa, J. L.; Hann, S. H.; Vining, C. B. L. Appl. Phvs. $1995,78,5474$.

(16) Nishida, I. Phys. Rev. B 1973, 7, 2710.

(17) Xia, Y. N.; Yang, P. D.; Sun, Y. G.; Wu, Y. Y.; Mayers, B.; Gates, B.; Yin, Y. D.; Kim, F.; Yan, Y. Q. Adv. Mater. 2003, 15, 353.

(18) Law, M.; Goldberger, J.; Yang, P. D. Ann. Rev. Mater. Res. 2004, $34,83$.

(19) Bavykin, D. V.; Lapkin, A. A.; Plucinski, P. K.; Friedrich, J. M.; Walsh, F. C. I. Phvs. Chem. B 2005, 109, 19422

(20) Lim, S. H.; Luo, J.; Zhong, Z.; Ji, W.; Lin, J. Inorg. Chem. 2005, 44, 4124.

(21) Liu, A.; Wei, M.; Honma, I.; Zhou, H. Anal. Chem. 2005, 77, 8068.

(22) Liu, A.; Wei, M.; Honma, I.; Zhou, H. Adv. Funct. Mater. 2006, 16, 371

(23) Gao, X. P.; Lan, Y.; Zhu, H. Y.; Liu, J. W.; Ge, Y. P.; Wu, F.; Song, D. Y. Electrochem. Solid-State Lett. 2005, 8, A26.

(24) Tokudome, H.; Miyauchi, M. Anqew. Chem. Int. Ed. 2005, 44, 1974.

(25) Khan, M. A.; Jung, H. T.; Yang, O. B. L.Phys. Chem. B 2006, 110, 6626.

(26) Liu, A.; Wei, M.; Honma, I.; Zhou, H. Anal.Chem. 2005, 77, 8068.

(27) Miao, L.; Ina, Y.; Tanemura, S.; Jiang, T.; Tanemura, M.; Kaneko, K.; Toh, S.; Mori, Y. Surf. Sci. 2007, 601, 2792.

(28) Miao, L.; Tanemura, S.; Jiang, T.; Tanemura, M.; Yoshida, K.; Tanaka, N.; Xu, G. Superlattices Microstruct. 2009, 46, 357.

(29) Mori, M.; Kumagai, Y.; Matsunaga, K.; Tanaka, I. Phvs. Rev. B 2009, 79, 144117-1

(30) Yoshida, K.; Miao, L.; Tanaka, N.; Tanemura, S. Nanotechnologv 2009, 20, 405709.

(31) Kurita, D.; Ohta, S.; Sugiura, K. L.Appl. Phvs. 2006, 100, 096105.

(32) Bavykin, D. V.; Friedrich, J. M.; Walsh, F. C. Adv. Mater. 2006, $18,2807$.

(33) Tang, H.; Prasad, K.; Sanjines, R.; Schmid, P. E.; Levy, F. L. Appl. Phvs. 1994, 75, 2042

(34) Wunderlich, W.; Ohta, H.; Koumoto, K. Phvsica B 2009, 404, 2202.

(35) Filipic, C.; Levstik, A.; Kutnjak, Z.; Umek, P. L. Appl. Phys. 2007, 101, 084308.

(36) Touloukian, Y. S.; Liley, P. E.; Saxena, S. C. Thermal Properties of Matter; IFI/PLENUM: New York, 1970; Vol. 3, pp 202-206.

(37) Venkatasubramanian, R.; Siivola, E.; Colpitts, T.; O'Quinn, B. Nature 2001, 413, 597.

(38) Zhao, X. B.; Ji, X. H.; Zhang, Y. H.; Zhu, T. J.; Tu, J. P.; Zhang, X. B. Appl. Phvs. Lett. 2005, 86, 062111

(39) Yang, R. G.; Chen, G.; Dresselhaus, S. Phys. Rev. B 2005, 72, 125418

(40) Ohta, H.; Kim, S.; Mune, Y.; Mizoguchi, T.; Nomura, K.; Ohta, S.; Nomura, T.; Nakanishi, Y.; Ikuhara, Y.; Hirano, M.; Hosono, H.; Koumoto, K. Nat. Mater. 2007, 6, 129.

(41) Riss, A.; Berger, T.; Grothe, H.; Bernardi, J.; Diwald, O.; Knozinger, E. Nano Lett. 2007, 7, 433.

AM100365Y 